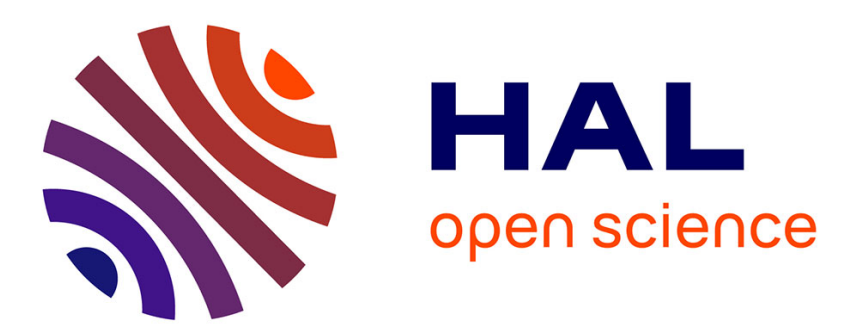

\title{
Introduction: Approche critique des transferts contemporains des modèles de développement territorial vers les Suds
}

Kirsten Koop, Anne-Laure Amilhat Szary

\section{- To cite this version:}

Kirsten Koop, Anne-Laure Amilhat Szary. Introduction: Approche critique des transferts contemporains des modèles de développement territorial vers les Suds. L'Information géographique, 2011, 75 (4), pp.6-14. 10.3917/lig.754.0006 . halshs-00694384

\section{HAL Id: halshs-00694384 \\ https://shs.hal.science/halshs-00694384}

Submitted on 4 May 2012

HAL is a multi-disciplinary open access archive for the deposit and dissemination of scientific research documents, whether they are published or not. The documents may come from teaching and research institutions in France or abroad, or from public or private research centers.
L'archive ouverte pluridisciplinaire $\mathbf{H A L}$, est destinée au dépôt et à la diffusion de documents scientifiques de niveau recherche, publiés ou non, émanant des établissements d'enseignement et de recherche français ou étrangers, des laboratoires publics ou privés. 


\title{
INTRODUCTION. APPROCHE CRITIQUE DES TRANSFERTS CONTEMPORAINS DES MODĖLES DE DÉVELOPPEMENT TERRITORIAL VERS LES SUDS
}

\author{
Kirsten Koop et Anne-Laure Amilhat \\ Armand Colin | L'Information géographique
}

\section{1/4 - Vol. 75 \\ pages 6 à 14}

ISSN 0020-0093

Article disponible en ligne à l'adresse:

http://www.cairn.info/revue-l-information-geographique-2011-4-page-6.htm

Pour citer cet article :

Koop Kirsten et Amilhat Anne-Laure, «Introduction. Approche critique des transferts contemporains des modèles de développement territorial vers les Suds »,

L'Information géographique, 2011/4 Vol. 75, p. 6-14. DOI : 10.3917/lig.754.0006

Distribution électronique Cairn.info pour Armand Colin.

(C) Armand Colin. Tous droits réservés pour tous pays.

La reproduction ou représentation de cet article, notamment par photocopie, n'est autorisée que dans les limites des conditions générales d'utilisation du site ou, le cas échéant, des conditions générales de la licence souscrite par votre établissement. Toute autre reproduction ou représentation, en tout ou partie, sous quelque forme et de quelque manière que ce soit, est interdite sauf accord préalable et écrit de l'éditeur, en dehors des cas prévus par la législation en vigueur en France. II est précisé que son stockage dans une base de données est également interdit. 


\title{
Introduction. Approche critique des transferts contemporains des modèles de développement territorial vers les Suds
}

\author{
Par Kirsten Koop, Anne-Laure Amilhat
}

Kirsten Koop, Anne-Laure Amilhat, Université Joseph-Fourier Grenoble - UMR PACTE

kirsten.koop@ujf-grenoble.fr ; anne-laure.amilhat@ujf-grenoble.fr

Bien que cela soit rarement discuté de manière explicite, le paradigme du développement des pays des Suds a définitivement changé. La vision moderniste, prônant le « rattrapage des retards des pays » dits « sous-développés » semble s'être effacée silencieusement, laissant la place à une nouvelle vision, propre à l'ère de la mondialisation : celle de l'injonction d'intégration des territoires dans les flux et réseaux mondiaux, surtout économiques et financiers (Koop, 2006). Pour la première fois depuis la décolonisation, cette vision du développement a valeur universelle : l'intégration aux réseaux est devenue comme une formule magique, aussi bien pour les Suds que pour l'Occident. Ce dernier, secoué par des crises diverses depuis la libéralisation des marchés, ne représente plus en conséquence le stade ultime du développement à atteindre tel que le postulait la théorie du take-off (Rostow, 1961).

\section{- Un nouveau paradigme}

Dans ce contexte, ce sont désormais les territoires à l'échelle infranationale qui sont considérés, par les chercheurs ainsi que par les organismes publics de l'aménagement des pays du Nord, comme les « points nodaux des processus de développement et de croissance » (Scott, Storper, 2003, 32) et le gabarit/l'échelle appropriée «d'action pour répondre aux inflexions mondiales » (Keating, Loughlin, 1997, 11). Ce changement de paradigme s'exprime dans les discussions académiques sur les dynamiques territoriales et le re-scaling, c'est-à-dire le redéploiement du pouvoir à des échelles subnationales (Keating, 2009 ; Brenner, 2004), mais aussi au travers des processus de décentralisation et de la mise en avant de régions souvent urbaines sur le plan de l'aménagement (comme le « Grand Paris » en France). L'émergence, dans l'hémisphère Sud, de régions et de lieux profitant, à une échelle infra-nationale, des chances de 
la mondialisation, n'a fait que valider l'universalité de cette nouvelle approche du développement (voir, par exemple, sa validation par le rapport sur le développement dans le monde de la Banque mondiale, 2009). Elle s'est traduite par la multiplication extraordinaire de modèles, concepts et stratégies durant ces dernières deux décennies, mettant tous les territoires au centre des préoccupations. «Intégration régionale », « clusters » (Porter, 2001), « systèmes productifs localisés » (Colletis et al., 1990), « réseaux innovateurs » (Camagni, 1991), « regionalisation » (Krätke, 1995), new regionalism (Keating and Loughlin, 1997 ; Mac Leod, 2001), « compétitivité systémique » régionale (Esser $e t$ al., 1996) « compétitivité territoriale », « attractivité territoriale », « spécificité territoriale » (Pecqueur) - la liste est longue. Même des concepts purement scientifiques et moins opérationnels de prime abord, comme ceux de ville globale (Sassen, 1991) ou de ville créative (Florida, 2002) semblent être devenus des leitmotivs pour les politiques de développement à toutes les échelles, au Nord tout comme aux Suds.

Cette universalisation de la vision du développement se traduit aussi bien par un changement d'échelle que par celui des stratégies à mettre en œuvre, ainsi que par la concomitance des défis du développement au Nord et aux Suds. On peut dès lors s'étonner que les modèles et stratégies politiquement dominants continuent d'émaner principalement du Nord pour être transmis vers les Suds par le biais de l'aide au développement - tout comme ce fut le cas dans le passé. Ce n'est que dans le monde altermondialiste et dans les marges institutionnelles qu'un véritable dialogue entre la société civile du Nord et des Suds et un début de co-construction de stratégies semble s'installer ${ }^{1}$.

La coopération internationale, quant à elle, semble ne pas avoir «perdu le nord » : les activités contemporaines des institutions internationales et organismes nationaux de coopération, influençant les devenirs des Suds depuis la décolonisation, reflètent parfaitement l'imposition du nouveau paradigme de développement aux Suds. Dans le sillage des Programmes d'Ajustement Structurel, le FMI et la Banque mondiale avaient déjà imposé la décentralisation aux Suds - condition conçue comme préalable au «développement » à l'ère de la mondialisation, à partir des années 1980. L'usage de termes équivalents cachait d'ailleurs souvent des réalités plus contrastées aux Suds, où les implications financières négatives des mesures de gouvernance échappaient aux occidentaux, séduits par les apports de la décentralisation dans leur quotidien (Osmont, 1998). Par la suite, le soutien à la gouvernance locale et à la capacitation des acteurs publics et de la société civile aux échelles infranationales sont devenus des domaines d'aide importants (voir, par exemple, le programme

1. Dont l'élargissement du débat sur l'après-développement ou l'approche participative (Perrin/Elson 2011) hors des cercles spécialistes témoigne. 
GOLD de l'Union européenne ou encore le Partenariat pour le Développement Municipal-PDM du Nouveau Partenariat pour le Développement de l'Afrique - NEPAD en Afrique de l'Ouest). Si l'idéologie occidentale contemporaine n'est pas transmise aux Suds via des programmes la servant explicitement, elle transcende la plupart des projets de coopération à travers leurs discours normatifs. Une révision des programmes contemporains montre qu'ils présupposent l'autonomie publique locale aussi bien que l'existence d'un développement économique local (voir, par exemple, l'Agenda 21, les Documents de stratégie pour la réduction de la pauvreté (DSRP) de la Banque mondiale, ou encore le Participatory Squatter Settlement Upgrading Programme (PSUP) de UNHabitat et leur application locale, telle que les articles de P.A. Landel et de S. Louargant, E. Matteudi et E. Roux les discutent.

Les progrès de développement sont désormais mesurés par l'intégration à la mondialisation. Aux indicateurs de développement habituels s'ajoutent les mesures des investissements directs étrangers, du taux d'exportation, de la libéralisation des marchés, de la mondialité des lieux (dont témoignent différents classements, comme le tableau des villes globales produit par le Globalisation and World Cities - GaWC, auxquels les grandes métropoles des Suds tentent de se rattacher). La désagrégation des données statistiques au niveau des régions est de plus en plus fréquente, comme le témoignent les derniers rapports du PNUD et de la Banque mondiale (cf. aussi l'article d'Ivan Samson dans ce numéro). Il va de soi que la production de ce type de statistiques à l'échelle mondiale a un impact fortement normatif.

Après tant de décennies d'échecs dans le transfert de modèles occidentaux vers les Suds, on peut se demander ce qui justifie cette ingérence au nouveau visage. Ce qui marche au Nord n'est pas nécessairement la solution appropriée pour les Suds - les échecs multiples et récurrents des projets de développement modernistes, les fameux «éléphants blancs », initiés par les agences occidentales d'aide au développement depuis la décolonisation, nous l'ont bien montré. La critique au transfert des modèles de développement occidentaux vers les pays des Suds date de plus d'un demi-siècle maintenant. Une des premières voix qui s'élevait fut celle de l'anthropologue Margareth Mead en 1955, mettant en garde contre des dommages psychiques et sociaux que l'aide occidentale au développement risquait de causer. Les critiques s'amplifièrent à partir des années 1970 avec l'émergence de la théorie de la dépendance (Franck, 1968 ; Lacoste, 1968). Dans le sillage de cette critique fondamentale aux rapports de pouvoir inégaux entre Nord et Suds, accusant le Nord d'être à l'origine même du « sous-développement » des Suds, se leva logiquement celle aux contenus et modes de l'aide internationale au développement. Les adeptes de la théorie de la dépendance plaidèrent pour une réorientation des stratégies de développement vers des démarches endogènes, fondées sur les ressources économiques et humaines internes. Le développement par le bas (bottom-up), participatif était 
supposé être plus approprié aux réalités locales et par là même plus prometteur (Stoehr/Taylor, 1981). Hélas, la mise en œuvre de ces recommandations au sein de l'aide internationale au développement n'a été que très ponctuelle. ${ }^{2}$

De nos jours, c'est le courant du post-colonialisme qui, dans le sillage de la déconstruction des discours et schémas de pensées coloniaux ${ }^{3}$ en œuvre aujourd'hui encore, critique non seulement les contenus de la soi-disant « coopération » internationale, mais la légitimité de l'ingérence en elle-même ${ }^{4}$. Quant au courant du post-développement, il refuse catégoriquement le système capitaliste et ainsi toute aide au « développement », considéré comme une impasse en soi (cf. Rahnema/Bawtree, 1997). Aucune de ces deux approches n'est réellement travaillée en France, où la "géographie du développement » semble d'ailleurs être tombée en déshérence faute d'avoir su reformuler à sa façon ce débat. Leur portée potentiellement très contestataire explique sans doute des réticences que les milieux académiques anglophones, plus cosmopolites, ont eu moins de mal à franchir, les avancées des études littéraires (cf. Bhaba 1990) ayant beaucoup influencé les méthodes en sciences sociales. Aussi, la critical development geography semble avoir intégré ces courants.

À quel point les modes de pensée académique occidentaux et les politiques dominantes de coopération se sont-ils ouverts aux critiques de ces différents courants ? Qu'ont-ils appris des difficultés, voire des échecs antérieurs des postures occidentales par rapport aux Suds? Qu'est-ce qui peut justifier, à l'ère de la globalisation, la continuation d'un regard du Nord dans les affaires des Suds ? Nous inscrivant dans les réflexions sur la circulation des concepts entre Nord et Suds, entamées dans le numéro spécial de L'Information géographique (1- 2011), nous proposons d'interroger ici de manière critique les transferts explicites ou implicites du paradigme territorial du Nord vers les Suds dans les nouveaux contextes décrits ci-dessus. Par définition liée à la spécificité des lieux, cette approche s'avère-t-elle plus adaptée aux réalités et spécificités des Suds que les approches antérieures ? Permet-elle d'éviter des fautes commises dans les projets de développement depuis des décennies, de mieux cerner les particularités des Suds, décrétées dans le passé comme facteurs du «sousdéveloppement » ? Ou s'agit-il, une fois de plus, de gommer toute différence en universalisant une idéologie ?

\footnotetext{
2. Ainsi, pour donner un exemple, l'organisme de coopération international principal en Allemagne, la Gesellschaft fuer technische Zusammenarbeit (GTZ) ne dépensait que $13 \%$ de son budget total pour des projets que l'on pourrait classifier « d'en bas » durant les années 1980 (Scholz 2004).

3. À la suite des travaux fondateurs d'Edward Said (L'Orientalisme, 1978) qui, à partir d'une analyse de ce que l'Occident disait de l'Orient islamique, mit à jour l'européo-centrisme du discours sur l'ailleurs, la démarche post-coloniale a été élargie du champ politique et économique à une approche épistémologique large.

4. L'on peut référer surtout à Chakrabarty (2000) qui critique «l'eurocentrisme méthodologique » et les « stratégies hégémoniques d'imposition » de l'Occident.
} 
Sans s'inscrire d'emblée dans l'un ou l'autre des courants théoriques critiques, les auteurs des trois articles de ce dossier essaient d'analyser quelques objets et modalités des transferts, tout en questionnant la «transférabilité » de ces objets. Tout comme ceux présentés dans le premier numéro, ces articles sont le fruit des réflexions collectives menées lors des journées d'études organisées par le groupe de recherche « Les territoires aux Suds » de l'UMR PACTE à Grenoble sur les référents de l'action publique et de la négociation socio-spatiale aux Suds.

Les articles réunis ici occupent un statut très particulier dans le paysage scientifique français, très peu ouvert aux approches critiques de l'aménagement et du développement. Ils ont pour point commun d'être écrit par des enseignantchercheurs qui sont amenés à intervenir régulièrement dans le champ de la coopération internationale. Mais ce sont deux pans de l'activité scientifique qui communiquent peu, l'expertise scientifique étant peu amenée au retour réflexif sur les interventions dans l'action publique aux Suds. On perçoit d'ailleurs dans ces textes combien ce retour sur soi est difficile.

À la fois par manque de temps et par crainte de ce risque, les praticiens du développement écrivent peu eux-mêmes. C'est pourquoi ces textes sont également précieux au-delà du contexte international, car les chercheurs porteurs des paradigmes post-développementalistes pratiquent rarement eux-mêmes les transferts qu'ils critiquent. Les auteurs réunis ici ont accepté la tâche délicate de distancier leur pratique, et de s'auto-appliquer des grilles de références construites pour autrui. Le résultat est particulièrement intéressant et ouvre certainement des pistes pour l'avenir.

\section{- Le transfert et la transférabilité du paradigme du " développement territorial » en question}

Faisons abstraction ici des discussions scientifiques sur le concept du «territoire », très propre aux sciences sociales françaises, et comprenons le modèle du «développement territorial » ici dans son sens large, désignant cette approche universalisée du développement qu'est l'injonction de l'intégration des espaces aux échelles infra-nationales et qui se distingue par son intérêt porté aux acteurs territoriaux, la territorialisation des actions, sa dimension participative, ainsi que par son approche systémique et multi-scalaire (Amilhat, Koop, 2010).

Il est intéressant d'interroger le sens et les conditions de sa diffusion dans les Suds en tant que nouvel objet de la coopération internationale. Ses objectifs semblent permettre de justifier le transfert, au-delà d'une croyance d'ordre purement théorique en la valeur universelle de l'approche territoriale dans le contexte de la mondialisation. Les stratégies par le bas, caractéristiques du 
modèle territorial, ont été revendiquées depuis trois décennies par le courant dépendantiste, et font aujourd'hui la doxa des approches occidentales de l'aménagement. Paradoxalement ou non, c'est à l'ère néo-libérale que les modes participatifs de conduite de projets localisées ont fait leur percée dans les programmes internationaux. Il s'agit de savoir si cette nouvelle forme d'aide au développement représente une bonne voie.

Les contributions de Sophie Louargant, Emmanuel Matteudi et Emmanuel Roux ainsi que celle de Pierre-Antoine Landel nous mettent en garde face à un optimisme trop rapide. En analysant, sous différents angles de vue, les modalités des transferts de compétences de développement territorial et leur réception locale, ils semblent plutôt confirmer le constat généralisé que la coopération pour le développement s'est enfoncée dans une aporie en imposant depuis le Nord des directives normatives sur des modes de faire participatifs ! Et ce dans un contexte qui a du mal à prendre en compte les valeurs, intentions et modes de faire des contreparties déjà à l'œuvre dans ce domaine, dans les Suds (Bueschel/Speich 2009).

S. Louargant, E. Matteudi et E. Roux analysent la spécificité de la position de l'expert à un tournant de l'action publique dans les Suds, celui d'une décentralisation dont les modalités se démocratisent enfin. C'est dans ce contexte que la coopération internationale s'est redéployée, tant au niveau international (comme le montre l'exemple de la multiplication des Agendas 21 au Maroc sous l'impulsion du PNUD), que bilatéral (avec de nouvelles priorités pour le Fonds de solidarité prioritaire - FSP) en matière d'aménagement du territoire. Les auteurs se présentent non seulement comme des chercheurs engagés dans l'action mais également comme des formateurs, s'étant donc retrouvés dans la position d'une transmission explicite. Pour ces derniers, l'expert qui prétend comprendre les territoires et leur diversité ne peut se cantonner à son rôle de « diffuseur » d'une pensée, ni même à celui de " passeur » de modalités d'action publique, il doit se faire « agenceur », à l'écoute des arrangements locaux qu'il contribue à faire évoluer. La nature ponctuelle des interventions extérieures, réduit néanmoins leur portée, et les chercheurs sont conscients de l'effet d'opportunité, pour les décideurs des Suds, de choisir un nouveau modèle pour capter des fonds de coopération, au risque de « zapper » parmi les références proposées par les différents bailleurs. D'où l'importance de pouvoir compter sur des relais formatifs pour ancrer les changements proposés dans la durée.

À travers un va-et-vient entre théorie et expérience personnelle pratique, PierreAntoine Landel analyse le transfert du modèle de «projet de territoire », opéré vers les pays du Maghreb à travers divers programmes du gouvernement français et de l'Union européenne, la DATAR ayant été impliquée comme acteur principal des transferts sur le terrain. Après un plaidoyer pour le développement 
territorial comme approche pertinente du développement dans les Suds - et donc sans mettre en question le sens et l'utilité du transfert en lui-même - il interroge sa pertinence et son efficacité, au regard des dynamiques territoriales locales. Son analyse met en lumière que la pré-condition indispensable à l'émergence du développement territorial qu'est l'autonomie des acteurs publics aux échelles subnationales, fait défaut. La capacité des acteurs territoriaux publics à intégrer les objets de la coopération se révèle être très limitée, faute de capacités humaines, de moyens financiers et d'autonomie décisionnelle. Au cours du montage des projets de territoire, ce déficit a même « rendu aléatoire la légitimité » des autorités publiques par rapport aux autres acteurs. L'auteur renvoie à un deuxième frein se situant dans les pratiques de coopération. Les modalités de transfert de ce modèle qui mise sur la participation ne prévoyaient pas systématiquement l'implication de la société civile, que ce soit du monde associatif ou de la chefferie coutumière.

Afin de sortir des impasses dans les pratiques actuelles, P.A. Landel renvoie à la nécessité de la « capitalisation », concept interrogeant le passage de l'expérience accumulée à partir d'une succession d'actions, à la construction de connaissances transmissibles entre les acteurs concernés. En proposant des pistes pour l'application concrète de ce concept dans les démarches de transfert $\mathrm{du}$ « développement territorial », il ouvre la voie vers un passage du « transfert » à une véritable co-construction de l'action publique - qui nécessiterait certes de profondes modifications encore dans la conception et la mise en œuvre des actions au sein de la coopération internationale.

Ivan Samson, passionné des chiffres et véritable artiste de la production de données territoriales, a répondu à notre sollicitation en revenant sur ses 20 ans de pratique d'analyse des dynamiques territoriales, menées à travers des études empiriques en ex-RDA, en Europe de l'Est, en Algérie et en Afrique de l'Ouest et du Centre. Travaillant sur la polarité urbaine, indicateur composite développé en sciences régionales européennes, son approche s'inscrit pleinement dans le paradigme du développement contemporain, qui suppose une corrélation positive entre urbanisation et développement (Banque mondiale, 2002, 2009). Son expérience de terrains si divers dans leurs réalités socio-économiques et politiques lui permet de discuter de manière circonspecte la transposabilité de cet indicateur et ainsi sa validité globale en tant qu'indicateur de développement à l'ère de la mondialisation. L'application globale de ce concept permet à l'auteur de relever les différences dans les processus de développement dans les différentes régions étudiées. Or les transferts Ouest-Est et surtout Nord-Suds causent des difficultés, aussi bien d'ordre méthodologique que conceptuelles. C'est justement la révélation et l'analyse de ces difficultés qui permettent à l'auteur d'apporter des éléments nouveaux au concept lui-même et d'enrichir son appareil méthodologique. Mais c'est surtout son constat concernant la validité globale de cet outil de mesure qui peut bouleverser : il affirme que 
cet instrument de mesure n'est finalement pas transposable du Nord vers les Suds, et notamment pas vers l'Afrique, les dynamiques urbaines étant fondamentalement différentes.

De la même façon que les textes réunis dans le $\mathrm{n}^{\circ}$ 1-2011 que nous avons coordonnés, ces articles nous renvoient directement à nous-mêmes : les auteurs avouent plus ou moins explicitement ne plus pratiquer et analyser l'aménagement aux Suds de la même façon avant et après la phase de coopération, voire de recherche, dont on peut dire qu'elle constitue une double épreuve, pour le destinataire comme pour le pourvoyeur du support d'aménagement. C'est au titre de son franchissement que le bilan se construit, positif si les positions de départ ont évolué, si la discussion sur la norme occidentale qui doit s'hybrider pour circuler permet en retour une évolution des paradigmes en usage chez nous d'une part, de nos pratiques de recherche d'autre part. Si, comme le propose P.-A. Landel, le territoire passe du statut d' « objet de recherche » à celui de «partenaire dans la recherche » avant d'accéder à celui de « sujet de recherche », alors la démarche de l'expert redevenu chercheur peut renouveler les pratiques de l'aménagement.

\section{$\boldsymbol{V}$ Bibliographie}

Amilhat. A., Koop K. (2010). « Le territoire comme acteur dans les approches internationales du développement ? Une analyse comparative », communication au colloque « Les territoires, acteurs du changement ? Quelle place pour les sciences de l'action dans l'ère territoriale », 7-8 décembre 2010, Grenoble.

Bhabha H. K. (dir.) (1990). Nation and Narration, New York, Routledge.

Banque mondiale (2009). Reshaping Economic Geography, World Development Report 2009, Washington DC, World Bank, 410.

Banque mondiale (2002). Systèmes de villes : l'urbanisation au service de la croissance et de la lutte contre la pauvreté. Stratégie de la Banque Mondiale pour les villes et les collectivités locales. Washington DC.

Brenner N. (2004). New State Spaces : Urban Governance and the Rescaling of Statehood, 0xfordNew York, Oxford University Press.

Bueschel H., Speich D. (éd.) (2009). Entwicklungswelten. Globalgeschichte der Entwicklungszusammenarbeit, Frankfurt a. Main, Campus.

Camagni R., (éd.) (1991). Innovation Networks : Spatial Perspectives, Londres, Belhaven Press.

Chakrabarty D. (2000). Provincializing Europe : Postcolonial Thought and Historical Difference, Princeton, NJ.

Colletis G., Courlet C., Pecqueur B., Winterhalter F. (éd.) (1990). Les systèmes industriels localisés. Politiques publiques et systèmes industriels localisés en France, Italie et RFA, Grenoble, IREPD.

Esser K., Hillebrand W., Messner D., Meyer-Stamer J. (éd.) (1996). Systemic competitiveness. New governance patterns for Industrial Development, Londres/Oregon : Routledge, Frank Cass. 


\section{Introduction}

Florida R. (2002). The Rise of the Creative Class. And How it's Transforming Work, Leisure and Everyday Life, New York, Basic Books.

Franck A.G. (1968). Le Développement du sous-développement : l'Amérique latine, Paris, F. Maspero.

Lacoste Y. (1968). Géographie du sous-développement, Paris, PUF.

Keating M., Loughlin J. (éd.) (1997). The Political Economy of Regionalism, Luxembourg, Centre Robert Schuman.

Keating M. (2009). « Rescaling Europe », Perspectives on European Politics and Society, 10 (1), p. $34-50$.

Krätke, S. (1995). « Globalisierung und Regionalisierung », Zeitschrift für Wirtschaftsgeographie 83, 207-221.

Koop K. (2007). « Les nouvelles fractures du monde et le défi pour la recherche sur les inégalités de développement », Cahiers du GEMDEV, $\mathrm{n}^{\circ}$ 31, p.183-196.

Mac Leod, G. (2001). «New Regionalism Reconsidered: Globalisation and the Remaking of Political Economic Space », International Journal of Urban and Regional Research 25, p. 804829.

Mead M. (1955). Cultural Patterns and Technical Change, New York, New American Library.

Osmont A. (1998). "La "governance" : concept mou, politique ferme », Annales de la Recherche Urbaine (80/81), p. 19-26.

Perrin M., Elson M.P. (2011). « Le droit à la ville. Cheminements géographique et épistémologique », L'information géographique, 75 (1), p. 15-36.

Porter M.E. (2001). « Regions and the new economics of competition », in Scott, A.J., editor, Global City Regions : Trends, Theory, Policy, 0xford, 0xford University Press, p. 139-157.

Rahnema M., Bawtree V. (éd) (1997). The Post-Development Reader, Zed Books.

Rostow W. (1960). The Stages of Economic Growth, Cambridge, Cambridge University Press.

Sassen S. (1991). The Global City : New York, London, Tokyo, Princeton, Princeton University Press.

Scholz F. (2004). Geographische Entwicklungsforschung. Methoden und Theorien, Berlin-Stuttgart, Gebrüder Bornträger.

Scott A.J., Storper M. (2003). « Regions, globalization, development », Regional Studies, p. 579593.

Stöhr W., Taylor D., (éd.) (1981) : Development from Above or Below ? The Dialectics of Regional Planning in Developing Countries, New York, John Wiley and Sons Ltd. 